\title{
Avant-propos
}

\section{Y. Soyer}

SQODF, Ancien AHU - Paris Diderot, Attaché à I'hôpital Pitié-Salpêtrière, Paris

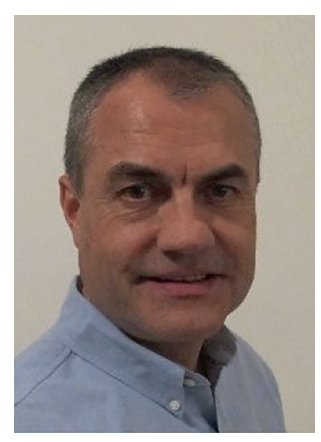

Tous les travaux et études qui sont menés méritent d'être partagés. La qualité des communications affichées présentées lors du congrès de la Société française d'orthopédie dento-faciale, à Biarritz, atteste de la progression régulière tant dans l'implication que du niveau de connaissance des participants.

La transmission du savoir passe d'une génération de cliniciens à l'autre et c'est très bien. La Revue d'Orthopédie Dento-Faciale existe depuis plus de 50 ans et participe à cette diffusion. Les volumes publiés sont élaborés pour des praticiens et par une équipe de praticiens qui ne demande qu'à s'étoffer. Je ne peux qu'inviter les lecteurs à venir la rejoindre. Après la publication de son numéro d'avril 2014, la rédaction de la Revue d'Orthopédie Dento-Faciale a souhaité un nouveau numéro consacré aux travaux de jeunes auteurs.

L'appel a été fait auprès des Internes et anciens Internes des hôpitaux et c'est avec un enthousiasme non dissimulé qu'ils nous présentent, pour certains, leurs premières publications.

Chloé Choukroune nous propose un guide clinique pour répondre à la situation du retard d'éruption dans le cadre de la mise en œuvre d'une thérapeutique adaptée.

Dans le but d'évaluer les effets sur l'évolution du parodonte, Bertrand Ducroz livre les résultats d'une étude analysant la modification de la hauteur coronaire lors des déplacements dentaires et plus spécifiquement en suivant les principes de Dwight Damon.

Charles Henry Fouquet expose un protocole d'interception démontrant l'intérêt de l'avulsion des canines temporaires dans le cadre de la mise en place des canines permanentes en situations ectopiques.

Afin d'éviter les effets iatrogènes des thérapeutiques conventionnelles d'expansion lors des disjonctions maxillaires rapides, Margaux Montigny propose I'utilisation raisonnée de mini-implants, associés ainsi à des appareils à appuis mixtes chez des patients à typologie parodontale fragile.

La gestion de la stabilité du sens transversal après expansion maxillaire pose régulièrement problème au clinicien et le comportement neuromusculaire est une des composantes de cette stabilisation ou de sa récidive. L'étude de Mathilde Tsang Tung contribue à démontrer l'importance de l'apport des contraintes 
masticatrices dans la correction transversale de l'occlusion.

Diane Zeitoun montre le réel intérêt qu'offre la chirurgie précoce. II ne faut donc pas oublier cette approche en présence d'une attente spécifique des patients, lors de thérapeutiques orthochirurgicales, et dans le cadre des échanges entre le patient, le chirurgien et l'orthodontiste.

Dans sa pratique quotidienne, l'orthodontiste peut avoir certaines difficultés dans sa décision thérapeutique de choisir un traitement avec ou sans chirurgie. Ines Dallel et son équipe nous proposent quelques axes de réflexion où la clinique, une fois de plus, révèle toute son importance.

Frédéric Haïm nous démontre que les mini-vis font partie des règles de bonne pratique, notamment dans la mise en place des canines incluses vestibulaires en technique linguale.
À travers des cas cliniques, Carine Ben-Younes apporte sa contribution dans le traitement des infraclusions antérieures en démontrant l'intérêt de la stimulation masticatoire pour une remise en fonction des secteurs antérieurs.

Enfin, la revue de presse est toujours présente grâce à Hélène Guiral-Desnoës.

Je saisis l'occasion pour remercier Alain Benauwt au nom de la rédaction de la Revue d'ODF et de tous les lecteurs. Alain a tenu la revue de presse avec assiduité et beaucoup de diligence pendant de nombreuses années et c'était toujours avec intérêt que nous lisions les « digests » qu'il traduisait pour nous. J'emploie à dessein le terme « digest » en clin d'œil vers Alain qui s'étonnait régulièrement de ne voir aucune publication d'auteur français collaborant avec les universités de différents pays...

Un très grand merci Alain et à tous ceux qui ont participé à l'élaboration de ce numéro. Excellente lecture.

Notre rédacteur en chef d'honneur Julien Philippe nous

a quittés le 13 juillet 2017. C'est avec une profonde tristesse

et une grande émotion que nous présentons nos sincères

condoléances à Maureen, son épouse, ainsi qu'à toute sa

famille. 